\title{
Aardwolf population diversity and phylogenetic positioning inferred using complete
} mitochondrial genomes

Westbury, Michael V.; De Cahsan, Binia; Dalerum, Fredrik; Noren, Karin; Hofreiter, Michael

Published in:

African Journal of Wildlife Research

DOI:

10.3957/056.049.0027

Publication date:

2019

Document version

Publisher's PDF, also known as Version of record

Document license:

CC BY-NC

Citation for published version (APA):

Westbury, M. V., De Cahsan, B., Dalerum, F., Noren, K., \& Hofreiter, M. (2019). Aardwolf population diversity and phylogenetic positioning inferred using complete mitochondrial genomes. African Journal of Wildlife Research, 49(1), 27-33. https://doi.org/10.3957/056.049.0027 


\section{Aardwolf Population Diversity and Phylogenetic Positioning Inferred Using Complete Mitochondrial Genomes}

Authors: Westbury, Michael V., Cahsan, Binia De, Dalerum, Fredrik, Norén, Karin, and Hofreiter, Michael

Source: African Journal of Wildlife Research, 49(1) : 27-33

Published By: Southern African Wildlife Management Association

URL: https://doi.org/10.3957/056.049.0027

BioOne Complete (complete.BioOne.org) is a full-text database of 200 subscribed and open-access titles in the biological, ecological, and environmental sciences published by nonprofit societies, associations, museums, institutions, and presses.

Your use of this PDF, the BioOne Complete website, and all posted and associated content indicates your acceptance of BioOne's Terms of Use, available at www.bioone.org/terms-of-use.

Usage of BioOne Complete content is strictly limited to personal, educational, and non - commercial use. Commercial inquiries or rights and permissions requests should be directed to the individual publisher as copyright holder.

BioOne sees sustainable scholarly publishing as an inherently collaborative enterprise connecting authors, nonprofit publishers, academic institutions, research libraries, and research funders in the common goal of maximizing access to critical research. 


\title{
Aardwolf population diversity and phylogenetic positioning inferred using complete mitochondrial genomes
}

\author{
Michael V. Westbury ${ }^{1,2 *}(\mathbb{D})^{\S}$, Binia De Cahsan ${ }^{3}$, Fredrik Dalerum ${ }^{4,5,6}(\mathbb{D})$, \\ Karin Norén ${ }^{5}$ \& Michael Hofreiter ${ }^{2}$ (iD), \\ ${ }^{1}$ Natural History Museum of Denmark, University of Copenhagen, Øster Voldgade 5-7, DK-1350 Copenhagen K, Denmark \\ ${ }^{2}$ Department of Mathematics and Natural Sciences, Evolutionary Adaptive Genomics, Institute for Biochemistry and Biology, \\ University of Potsdam, Karl-Liebknecht-Str. 24-25, 14476 Potsdam, Germany \\ ${ }^{3}$ Department of Mathematics and Natural Sciences, Evolutionary Biology/Systematic Zoology, Institute for Biochemistry and Biology, \\ University of Potsdam, Karl-Liebknecht-Str. 24-25, 14476 Potsdam, Germany \\ ${ }^{4}$ Research Unit of Biodiversity (UO-CSIC-PA), Mieres Campus, University of Oviedo, 33600 Mieres, Asturias, Spain \\ ${ }^{5}$ Department of Zoology, Stockholm University, Stockholm, Sweden \\ ${ }^{6}$ Mammal Research Institute, Department of Zoology and Entomology, University of Pretoria, Hatfield, Pretoria, 0002 South Africa
}

Received 17 May 2018. To authors for revision 27 June 2018. Accepted 6 November 2018

\begin{abstract}
The aardwolf (Proteles cristata) is a unique, insectivorous species of the family Hyaenidae. It occupies a disjunct range in eastern and southern Africa and is possibly a remaining member of a historical dog-like hyaena clade. Although both local and global population status and trends are largely unknown, aardwolves are not uncommon in arid grasslands and the IUCN conservation status of Least Concern appears to be justified. However, they are one of the least studied of the four hyaena species, and we have scarce information about the genetic structure of aardwolf populations. Here, we present the first complete aardwolf mitochondrial genomes and provide novel information about aardwolf evolutionary origins and genetic structure within a single population. Through the investigation of complete mitochondrial genomes from five individuals from a single population within South Africa, we find the mitochondrial diversity of this population to be neither particularly high nor low compared to a number of other mammalian species. Moreover, we also provide additional evidence towards the basal position of the aardwolf within Hyaenidae with a divergence time of $13.0 \mathrm{Ma}(95 \% \mathrm{Cl} 10.1-16.4 \mathrm{Ma})$ from all other extant hyaena species.
\end{abstract}

Keywords: mitochondria, aardwolf, hyaena, genetic diversity, phylogenetics.

\section{INTRODUCTION}

The Hyaenidae, a family within the order Carnivora, is believed to have arisen approximately 25 million years ago (Ma) during the Late Oligocene (Werdelin \& Solounias, 1991). This family was once highly diverse and at the peak of its diversity, during the Late Miocene, consisted of more than 80 species across Europe, Asia, Africa and North America (Werdelin \& Solounias, 1991). However, in contrast to its past diversity, the family now comprises only four extant morphologically and ecologically distinct genera, each of which consists of only a single species. These extant lineages have been molecularly estimated to have arisen maximally $\sim 15 \mathrm{Ma}$ (Koepfli et al., 2006) and

*To whom correspondence should be addressed. E-mail: mvwestbury@gmail.com may therefore be a subclade within the Hyaenidae which consists of many more extinct basal fossil lineages. These genera are made up of the bone cracking Crocuta crocuta (spotted hyaena), Hyaena hyaena (striped hyaena) and Parahyaena brunnea (brown hyaena) and, the clear outlier within the extant species, the insectivorous Proteles cristata (aardwolf) (Koepfli et al., 2006). In addition to being the only non-bone cracking hyaena species alive today, the aardwolf is morphologically more similar to canids and is believed to be a morphologically primitive hyaena (Werdelin \& Solounias, 1991).

The aardwolf has a discontinuous distribution across Africa and occurs in two distinct areas separated by approximately $1500 \mathrm{~km}$ : east and northeastern Africa and southern Africa. This distribution pattern is believed to be due to the distribution of their main food source, termites of 
the genus Trinervitermes which are entirely absent from the central African woodlands (Coe \& Skinner, 1993; Kingdon et al., 2013). Despite a general lack of abundance data, the aardwolf is believed to have stable population numbers and is listed as Least Concern by the International Union for Conservation of Nature (IUCN) (Green, 2015).

Despite previous genetic work looking into the phylogenetic positioning of the aardwolf within the Hyaenidae (Koepfli et al., 2006), to date no complete mitochondrial genome has been published. In addition, there is a general lack of data on its genetic structure and variation, both within and between populations. Such data have been highlighted as a research priority for the southern parts of the species' range (deVries et al., 2016). Here, we present not only a novel aardwolf mitochondrial genome but also a first glimpse into the population structure and the genetic variation of the aardwolf through investigation of the mitochondrial genomes of five individuals from a single population within South Africa. Moreover, using these mitochondrial genomes, we provide additional evidence as to the aardwolf's phylogenetic positioning within the extant Hyaenidae family tree.

\section{MATERIALS AND METHODS}

We used skin tissue samples collected from five aardwolf captured for an ecological research project on Benfontein Game Reserve outside of Kimberley, central South Africa $\left(28^{\circ} 52^{\prime} \mathrm{S}, 24^{\circ} 50^{\prime} \mathrm{E}\right)$ (Marneweck et al., 2015). The reserve covers approximately 11400 ha and consists mainly of dry Karoo shrubland, arid grasslands and Kalahari thornveld (Dalerum et al., 2017). The animals were captured from 2008 to 2012 and resided in neighbouring home ranges (Marneweck et al., 2015). Captures were carried out under a permit from the animal care and use committee of the University of Pretoria (EC031-07) and permits from the provincial government in the Northern Cape (FAUNA 846/2009, FAUNA 847/2009). Aardwolves were immobilized by remote injection with $36.0 \mathrm{mg}$ ketamine hydrochloride and $0.6 \mathrm{mg}$ medetomidine hydrochloride using a $\mathrm{CO}_{2}$-powered dart gun (Kotze et al., 2012). All immobilizations were carried out by personnel registered by the South African Veterinary Council. Genetic samples consisted of a small piece of skin collected at the ear tip. Samples were stored in $95 \%$ ethanol at $-20^{\circ} \mathrm{C}$ until analyses.

We extracted DNA from the tissue samples using a Zymo Genomic DNA Clean \& Concentra- tor $^{\circledast}$ extraction kit (Zymo Research, U.S.A.), following the manufacturer's protocol. DNA extracts were built into Nextera Illumina sequencing libraries (Inqaba Biotec, Pretoria) and were sequenced on an Illumina Nextseq 500 at the University of Potsdam, Germany, using $2 \times 150$ bp paired-end reads. We then used Cutadapt v1.4 (Martin, 2011) to trim Illumina adapter sequences from the raw reads and remove reads shorter than $30 \mathrm{bp}$. Paired end reads with overlapping regions were merged using FLASH v1.2.10 (Magoĉè \& Salzberg, 2011). As there was no complete aardwolf mitochondrial genome to use as a mapping reference, we first constructed one from a single individual (Aardwolf15). We randomly subsampled 40 million merged reads from Aardwolf15 using seqtk ( $\mathrm{Li}, 2012)$ and then removed PCR duplicates using Prinseq (Schmieder \& Edwards, 2011). The remaining reads were treated as single end and underwent an iterative mapping approach using MITObim v1.8 (Hahn et al., 2013), a wrapper script for the Mira v4.0.2 (Chevreux et al., 1999) assembler following an approach similar to Westbury et al. (2017). We implemented MITObim using default parameters apart from mismatch values, where we used values from $0 \%$ to $3 \%$. Six independent runs in total were performed using two different extant hyaena reference bait sequences; Crocuta crocuta (Genbank accession JF8943 77.1) and Hyaena hyaena (Genbank accession NC_020669.1). Mira output maf files were converted to sam files and visualized using Geneious v9.0.5 (Kearse et al., 2012). We constructed initial mismatch value consensus sequences from all six assemblies using a minimum read coverage of $50 x$, ignoring gaps and used a $75 \%$ base call threshold. We then aligned these six sequences using Mafft v7.271 (Katoh \& Standley, 2013) and built a final consensus sequence by ignoring gaps and implementing a $75 \%$ base call threshold in Geneious. An automatic annotation of this final consensus sequence was performed using MITOS (Bernt et al., 2013) and more precise manual annotations were performed using Geneious.

The constructed mitochondrial genome then served as a reference sequence for subsequent mitochondrial mapping analyses. We mapped the trimmed and merged reads from our remaining four individuals to the reconstructed reference sequence with BWA v0.7.15 (Li \& Durbin, 2009), using the mem algorithm and default parameters and parsed the mapped files using Samtools v1.3.1 ( $\mathrm{Li}$ et al., 2009). The consensus sequences 
were constructed utilizing the consensus base call method and only considering reads of mapping quality greater than 25 and bases of phred quality scores greater than 25 with ANGSD v0.913 (Korneliussen et al., 2014).

We then aligned our aardwolf reference mitochondrial genome with a number of mitochondrial genomes from members within Feliformia (Supplementary Table S1) using Mafft. Next, we manually excluded the control region from further analyses due to its high variability that can lead to difficulties in a confident and meaningful alignment between species in this region. We then manually annotated the alignment for tRNAs, rRNAs, and protein coding regions to be used as input for Partitionfinder2 (Lanfear et al., 2017), which was used to group each mitochondrial genomic feature into partitions and then find the optimal substitution model for said partition (Supplementary Table S2). Using this alignment, we constructed a phylogenetic tree using BEAST v1.8.4 (Drummond \& Rambaut, 2007) on the Cipres server (Miller et al., 2010) specifying the partitions and substitution models as determined via Partitionfinder2, an uncorrelated lognormal relaxed clock model, and a birth-death speciation process (Gernhard, 2008). We further specified five exponentially distributed fossil calibration points (Supplementary Table S3) in the phylogeny based on those used in previous phylogenetic dating analyses (Eizirik et al., 2010; Paijmans et al., 2017). We ran the Markov Chain Monte Carlo (MCMC) chain for 10 million generations, sampling every 10000 generations, and assessed convergence of the posteriors (ESS> 200) using Tracer v1.6 (Rambaut et al., 2014). We extracted the maximum clade credibility tree with node heights scaled to the median of the posterior sample. Furthermore, in order to investigate the utility of single mitochondrial genes for a dated phylogenetic analysis, we repeated the above approach using the three most intraspecifically variable genes from the aardwolf dataset, COX1, Cytb and NAD3, independently and with all three genes combined as one dataset.

We also aligned our five complete aardwolf mitochondrial genomes using Mafft and constructed a median joining haplotype network using PopART (Leigh \& Bryant, 2015). From the same alignment, we calculated the mitochondrial diversity of the aardwolf by calculating the overall mean pairwise distances $(\mathrm{k})$, treating gaps and missing data as complete deletions using MEGA6 (Tamura et al., 2013). This $k$ value was then compared against previously published values from a number of other mammalian species (Westbury et al., 2018). Furthermore, in order to investigate other regions that may be useful in future phylogeographic studies of the aardwolf, we calculated the total and average number of variable regions in each protein coding gene and the control region. To make results comparable between different regions, we further calculated the average number of variable positions per site within each region (Supplementary Table S4).

\section{RESULTS}

We produced five complete aardwolf mitochondrial genomes (Genbank accession codes: MH662441-MH662445) using an initial iterative mapping approach of one individual followed by subsequent mapping of four other individuals to the iteratively mapped individual. The mitochondrial genome of Aardwolf15 contained 17159 bp, all 13 protein-coding genes with expected open reading frames, 22 transfer RNA genes, and two ribosomal RNA genes found within a typical vertebrate mitochondrial genome. We interpret these results as support for our data representing a correct and complete aardwolf mitochondrial genome. We found support for a basal placement of the aardwolf within the Hyaenidae, with a similar but slightly deeper mean divergence time of $13.0 \mathrm{Ma}(\mathrm{Cl} 10.1-16.4 \mathrm{Ma})$ compared to previous findings (10.6 Ma, $(95 \% \mathrm{Cl} 7.3-15.0 \mathrm{Ma}$, Koepfli et al., 2006). We placed Proteles with high confidence (posterior probability of 1) as a sister clade containing the bone crushing hyaena genera (Parahyaena, Hyaena and Crocuta) (Fig. 1).

When using the three genes Cytb, COX1, and NAD3, in a single combined phylogenetic analysis, we recovered a divergence time of $12.5 \mathrm{Ma}$ (95\% Cl 8.9-16 Ma). When using just the COX1 gene, we recovered a divergence time of $10.6 \mathrm{Ma}$ (95\% Cl 6.6-14.9 Ma). However, even after running the BEAST analysis for 100 million generations, neither of the NAD3 and Cytb datasets reached convergence and therefore, no results were obtained for these genes individually.

The haplotype network analysis showed three different haplotypes, defined as at least one substitution within the complete mitochondrial genome, that were fairly distant from one another (between 31 to 50 substitutions between haplotypes). However, we did find one haplotype to be shared between three individuals, Aardwolf08, 09 


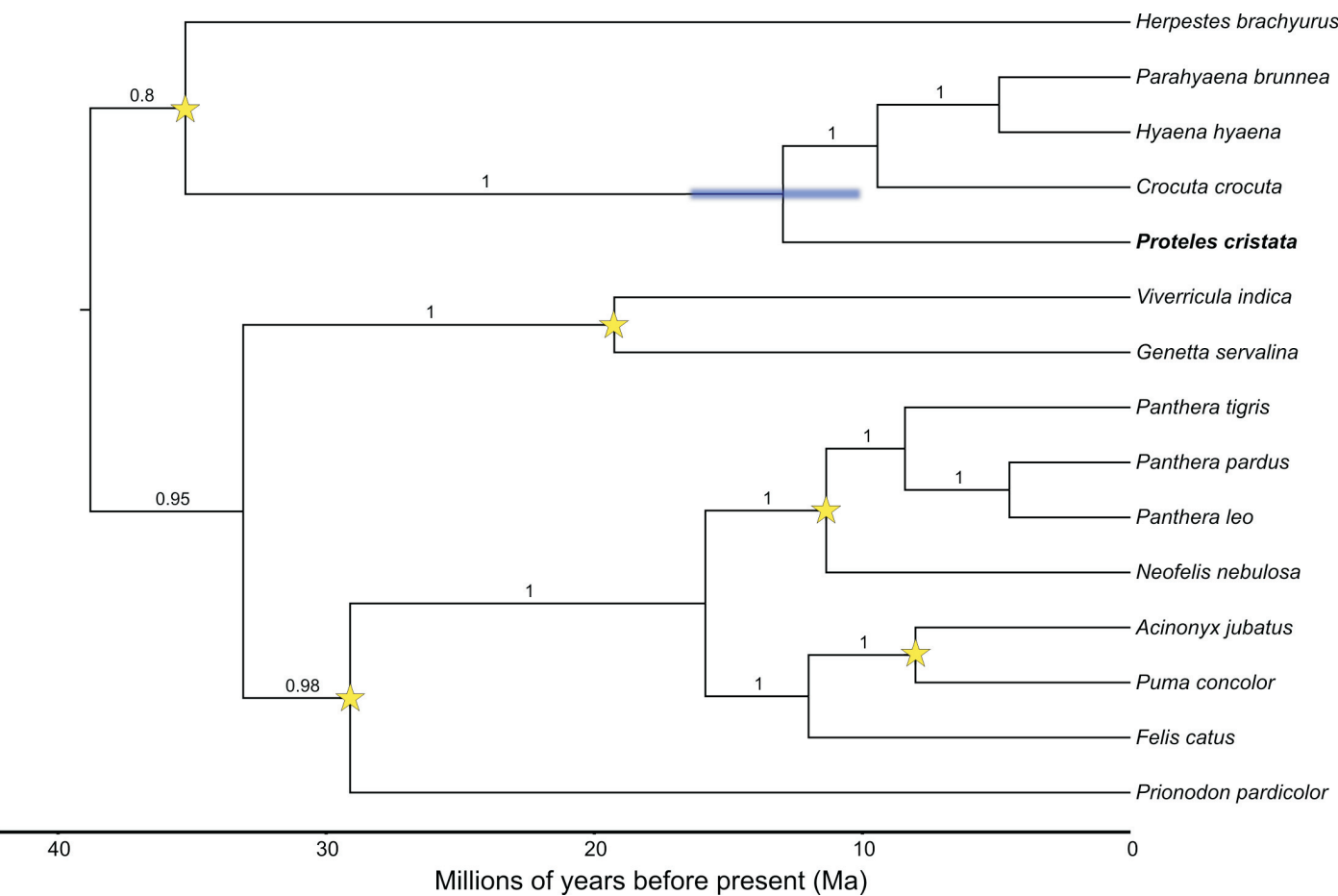

Fig. 1. Molecularly dated Bayesian phylogenetic tree constructed using the complete mitochondrial genomes of all extant Hyaenidae and various other members of the Feliformia suborder. Numbers on branches represent posterior probability support values, stars represent the branch nodes constrained by fossil calibration points and the light purple bar represents the $95 \%$ credibility interval for the divergence time of Proteles from the other extant hyaena genera. $X$ axis represents time in millions of years.

and 15 (Fig. 2). Finally, we found the mitochondrial diversity (k) of this population to be 31 (Fig. 3), placing it in an intermediate position between the other species included in this comparison. This diversity estimate was 7.75 times higher than the lowest in the comparison (the brown hyaena) and 6.12 times lower than the highest in this comparison (the chimpanzee (Pan troglodytes)).

We found 17 variable positions within the control region, making it the mitochondrial region with the highest total number of variable positions. The control region also had the highest average number of variable positions per site $(0.0182)$. All protein coding gene regions had between 1 and 9 variable positions and an average number of variable positions of between 0.0010 and 0.0145 per site (Supplementary Table S4).

\section{DISCUSSION}

Through the construction of five mitochondrial genomes, we provide a first glimpse into the genetic diversity of the aardwolf. We also provide additional evidence for the phylogenetic positioning of this last remaining dog-like hyaena species.
The mitochondrial genetic diversity was neither high nor low when compared to a number of other

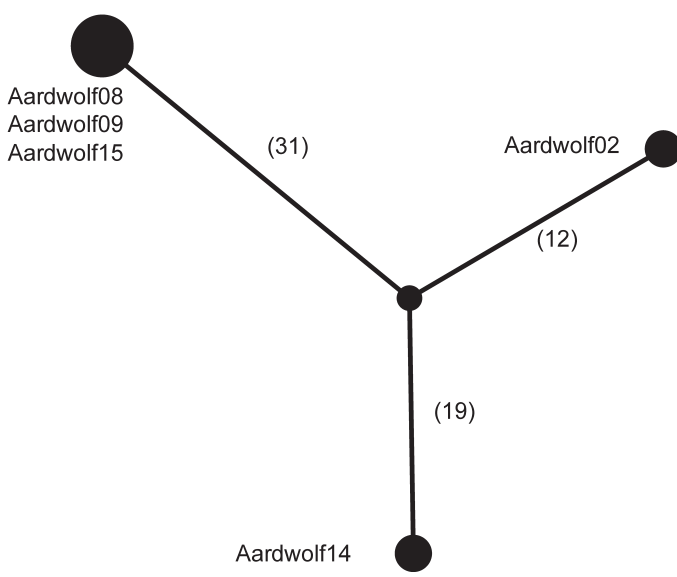

Fig. 2. Median joining haplotype network constructed using the complete mitochondrial genomes of the five aardwolf individuals included in the study. Filled black circles represent individual haplotypes, sizes of the circles represent the number of individuals per haplotype and numbers on the branches represent the number of substitutions between haplotypes. 


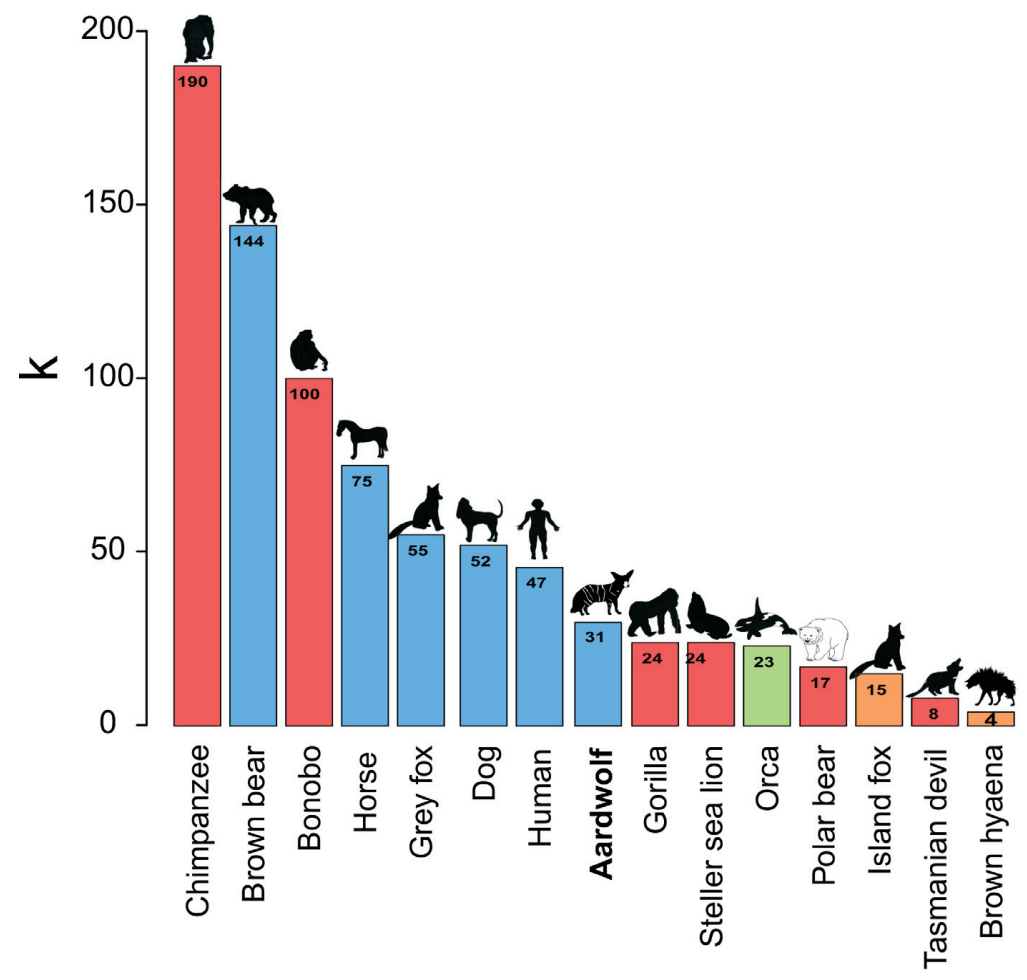

Fig. 3. Mitochondrial diversity comparisons between a number of other mammalian species as seen in Westbury et al., (2018). k represents the average number of substitutions expected between two randomly selected individuals of the same species, bar colours represent conservation status (red, endangered/threatened; orange, near threatened; blue, least concern; green, unavailable) according to the IUCN. Figure modified from Westbury et al., (2018) with permission.

species of varying IUCN conservation levels. This result is unsurprising and is in concordance with the aardwolf's listing of Least Concern by the IUCN (Green, 2015). Furthermore, as this dataset represents only a single population, one would assume the mitochondrial diversity to be higher when considering the species as a whole as new populations should present new haplotypes, especially in the aardwolf since it has a disjunct range (Coe \& Skinner, 1993; Kingdon et al., 2013). This assumption is further reinforced by the fact that we found three haplotypes that were relatively distant from one another within a sampling of just five individuals from one location. However, three individuals shared a single mitochondrial haplotype. This result may have arisen by chance in unrelated individuals due to the mitochondrial genome being inherited as a single locus, which can be inherited without any novel substitutions for many generations. However, it could also have arisen due to sampling of maternally related individuals as we sampled individuals with neighbouring home ranges, although we point out that we have scarce information regarding aardwolf dispersal behaviour.

Interestingly, the aardwolf has a lot more mitochondrial diversity when compared to its family relative, the brown hyaena, which showed a mitochondrial diversity $k$ value of only four across the entire species range as opposed to the aardwolf with a $\mathrm{k}$ value of 31 within just a single population. The low genetic diversity in the brown hyaena has been explained by a recent rapid genetic bottleneck (Westbury et al., 2018). However, as the genetic history of the aardwolf is currently unknown, more research, preferably with the help of nuclear genomes, would be needed to better explain the mitochondrial genetic diversity level found within this study. Current results, however, do not suggest the aardwolf has undergone such a bottleneck.

Confident phylogenetic placement of the aardwolf has met with difficulties in the past (Werdelin \& Solounias, 1991; Jenks \& Werdelin, 1998). Morphologically based phylogenetics were problematic for the aardwolf as, despite retaining many 
morphologically primitive characteristics, the aardwolf has a high number of uniquely derived morphological characteristics. One such example is dentition. Dentition is usually a valuable characteristic for morphology based phylogenetic analyses, however, due to the highly reduced dentition of the aardwolf, these comparative analyses were not possible when considering the placement of the aardwolf within the family Hyaenidae (Werdelin \& Solounias, 1991). Molecular data, on the other hand, has the ability to resolve this relationship as seen in previous studies (Jenks \& Werdelin, 1998; Koepfli et al., 2006). When using complete mitochondrial sequences, we found support for previous findings but with a slightly deeper mean divergence estimate. Furthermore, we found additional support when using subsampled datasets of either three protein coding genes Cytb, COX1, and NAD3 combined or the COX1 gene on its own. This result was not unexpected as the mitochondrial genome is inherited maternally as a single locus so, if enough information is present in the subsampled region, phylogenetic results are expected to be similar. However, in contrast to the COX1 gene alone having sufficient data to reconstruct a molecularly dated phylogeny, the single gene analyses of NAD3 and Cytb datasets did not reach convergence. This could indicate that the data contained in these regions were not sufficient for the current phylogenetic analysis. This could either be due to too few nucleotide positions or, more probably, too few variable positions within these regions between the selected species. Taken together, these results show that even when using a considerably subsampled section (3015 bp (19.4\%) or $1538 \mathrm{bp}(9.9 \%))$ of the mitochondrial genome (15 $533 \mathrm{bp)} \mathrm{alignment,} \mathrm{robust} \mathrm{results} \mathrm{can} \mathrm{still} \mathrm{be}$ obtained. However, it should be noted that as the amount of data in the analysis decreased, the credibility intervals increased, which should also be taken into consideration when selecting markers for future phylogenetic studies.

The convergent results from the current study together with those of previous studies, gives us confidence in both the phylogenetic placement and estimated divergence time of the aardwolf as even when different genetic markers, fossil calibration points and comparative species were used, the same results arose. In addition, we found high levels of variation within the control region, COX1 and NAD3 within just this single population of aardwolf, indicating that these regions may provide sufficient information to uncover phylogeographic structure within the aardwolf even when complete mitochondrial genomes are not available.

\section{ACKNOWLEDGEMENTS}

This work was supported by the European Research Council (consolidator grant GeneFlow \# 310763 to M.H), National Research Foundation (NRF, South Africa, various grants awarded to F.D., Elissa Cameron and Nigel Bennett), and the Spanish Ministry of Economy and Competitiveness (MINECO, RYC-2013-14662 to F.D.). The ecology division of the De Beers Consolidated Mines (Ltd) kindly allowed us to conduct the aardwolf study on Benfontein Nature Reserve. Finally, we would like to thank Armanda Bastos for the use of her laboratory facilities at the University of Pretoria, South Africa.

\section{${ }^{5}$ ORCID iDs}

M.V. Westbury:

F. Dalerum:

orcid.org/0000-0003-0478-3930

M. Hofreiter: orcid.org/0000-0001-9737-8242 orcid.org/0000-0003-0441-4705

\section{REFERENCES}

Bernt, M., Donath, A., Jühling, F., Externbrink, F., Florentz, C., Fritzsch, G., Pütz, J., Middendorf, M. \& Stadler, PF. (2013). MITOS: improved de novo metazoan mitochondrial genome annotation. Molecular Phylogenetics \& Evolution, 69, 313-319.

Chevreux, B., Wetter,T. \& Suhai, S., et al. (1999). Genome sequence assembly using trace signals and additional sequence information. In German Conference on Bioinformatics. Vol. 99: Heidelberg; pp. 45-56.

Coe, M.J. \& Skinner, J.D. (1993). Connections, disjunctions and endemism in the eastern and southern African mammal faunas. Transactions of the Royal Society of South Africa, 48, 233-255.

Dalerum, F., de Vries, J.L., Pirk, C.W.W. \& Cameron, E.Z. (2017). Spatial and temporal dimensions to the taxonomic diversity of arthropods in an arid grassland savannah. Journal of Arid Environments, 144, 21-30.

deVries, L., Marneweck, D., Dalerum, F., Mills, M.G.L., Yarnell, R., Sliwa, A. \& Do Linh San, E. (2016). A conservation assessment of Proteles cristata. In M.F. Child, L. Roxburgh, E. Do Linh San \& H.T. Davies-Mostert (Eds), The Red List of mammals of South Africa, Swaziland and Lesotho. South Africa: South African National Biodiversity Institute and the Endangered Wildlife Trust.

Drummond, A.J. \& Rambaut, A. (2007). BEAST: Bayesian evolutionary analysis by sampling trees. BMC Evolutionary Biology, 7, 214.

Eizirik, E., Murphy, W.J., Koepfli, K-P., Johnson, W.E., Dragoo, J.W., Wayne, R.K. \& O'Brien, S.J. (2010). Pattern and timing of diversification of the mamma- 
lian order Carnivora inferred from multiple nuclear gene sequences. Molecular Phylogenetics \& Evolution, 56, 49-63.

Gernhard, T. (2008). The conditioned reconstructed process. Journal of Theoretical Biology, 253, 769-778.

Green, D.S. (2015). Proteles cristata. The IUCN Red List of Threatened Species 2015: e. T18372A45195681. Retrieved from:

http://dx.doi.org/10.2305/IUCN.UK.2015-2.RLTS.T1 8372A45195681.en

Hahn, C., Bachmann, L. \& Chevreux, B. (2013). Reconstructing mitochondrial genomes directly from genomic next-generation sequencing reads-a baiting and iterative mapping approach. Nucleic Acids Research, gkt371.

Jenks, S. \& Werdelin, L. (1998). Taxonomy and systematics of living hyaenas (family Hyaenidae). Gland, Switzerland: IUCN/SSC Hyaena Specialist Group.

Katoh, K. \& Standley, D.M. (2013). MAFFT multiple sequence alignment software version 7: improvements in performance and usability. Molecular Biology \& Evolution, 30, 772-780.

Kearse, M., Moir, R., Wilson, A., Stones-Havas, S., Cheung, M., Sturrock, S., Buxton, S., Cooper, A., Markowitz, S., Duran, C., et al. (2012). Geneious Basic: an integrated and extendable desktop software platform for the organization and analysis of sequence data. Bioinformatics, 28, 1647-1649.

Kingdon, J., Happold, D., Butynski, T., Hoffmann, M., Happold, M. \& Kalina, J. (2013). Mammals of Africa. London: A\&C Black.

Koepfli, K-P., Jenks, S.M., Eizirik, E., Zahirpour, T., Van Valkenburgh, B. \& Wayne, R.K. (2006). Molecular systematics of the Hyaenidae: relationships of a relictual lineage resolved by a molecular supermatrix. Molecular Phylogenetics \& Evolution, 38, 603-620.

Korneliussen, T.S., Albrechtsen, A. \& Nielsen, R. (2014). ANGSD: Analysis of next generation sequencing data. BMC Bioinformatics, 15, 356.

Kotze, R., Bennett, N.C., Cameron, E.Z., Low de Vries, J., Marneweck, D.G., Pirk, C.W.W. \& Dalerum, F. (2012). Temporal patterns of den use suggest polygamous mating patterns in an obligate monogamous mammal. Animal Behavior, 84, 1573-1578.

Lanfear, R., Frandsen, P.B., Wright, A.M., Senfeld, T. \& Calcott, B. (2017). PartitionFinder 2: new methods for selecting partitioned models of evolution for molecular and morphological phylogenetic analyses. Molecular Biology \& Evolution, 34, 772-773.

Leigh, J.W. \& Bryant, D. (2015). POPART: full-feature software for haplotype network construction. Methods in Ecology and Evolution, 6, 1110-1116.

$\mathrm{Li}, \mathrm{H}$. (2012). seqtk Toolkit for processing sequences in FASTA/Q formats. Retrieved from: https://github.com/lh3/seqtk
Li, H. \& Durbin, R. (2009). Fast and accurate short read alignment with Burrows-Wheeler transform. Bioinformatics, 25, 1754-1760.

Li, H., Handsaker, B., Wysoker, A., Fennell, T., Ruan, J., Homer, N., Marth, G., Abecasis, G. \& Durbin, R., 1000 Genome Project Data Processing Subgroup. (2009). The sequence alignment/map format and SAMtools. Bioinformatics, 25, 2078-2079.

Magoĉè, T. \& Salzberg, S.L. (2011). FLASH: fast length adjustment of short reads to improve genome assemblies. Bioinformatics, 27, 2957-2963.

Marneweck, D., Cameron, E.Z., Ganswindt, A. \& Dalerum, F. (2015). Behavioural and endocrine correlates to the aardwolf mating system. Mammalian Biology - Zeitschrift für Säugetierkunde, 80, 31-38.

Martin, M. (2011). Cutadapt removes adapter sequences from high-throughput sequencing reads. EMBnet. journal, 17, 10-12.

Miller, M.A., Pfeiffer, W., \& Schwartz, T. (2010). Creating the CIPRES Science Gateway for inference of large phylogenetic trees. In 2010 Gateway Computing Environments Workshop (GCE). pp. 1-8.

Paijmans, J.L.A., Barnett, R., Gilbert, M.T.P., ZepedaMendoza, M.L., Reumer, J.W.F., de Vos, J., Zazula, G., Nagel, D., Baryshnikov, G.F., Leonard, J.A., et al. (2017). Evolutionary history of saber-toothed cats based on ancient mitogenomics. Current Biology, 27, 3330-3336.

Rambaut, A., Suchard, M.A., Xie, D. \& Drummond, A.J. (2014). Tracer v1. 6, http://beast.bio.ed.ac.uk

Schmieder, R. \& Edwards, R. (2011). Quality control and preprocessing of metagenomic datasets. Bioinformatics, 27, 863-864.

Tamura, K., Stecher, G., Peterson, D., Filipski, A. \& Kumar, S. (2013). MEGA6: molecular evolutionary genetics analysis version 6.0. Molecular Biology \& Evolution, 30, 2725-2729.

Werdelin, L. \& Solounias, N. (1991). The Hyaenidae: taxonomy, systematics and evolution. Fossils and Strata, 30, 1-104.

Westbury M., Baleka S., Barlow A., Hartmann S., Paijmans J.L.A., Kramarz A., Forasiepi A.M., Bond M., Gelfo J.N., Reguero M.A., et al. (2017). A mitogenomic timetree for Darwin's enigmatic South American mammal Macrauchenia patachonica. Nature Communications, 8, 15951.

Westbury, M.V., Hartmann, S., Barlow, A., Wiesel, I., Leo, V., Welch, R., Parker, D.M., Sicks, F., Ludwig, A., Dalén, L. \& Hofreiter, M. (2018). Extended and continuous decline in effective population size results in low genomic diversity in the world's rarest hyena species, the brown hyena. Molecular Biology \& Evolution, 35, 1125-1237.

Responsible Editor: S.M. Miller 